\title{
Lightweighting design optimisation for additively manufactured mirrors
}

\author{
Carolyn Atkins, William Brzozowski, Naomi Dobson, \\ Maria Milanova, Stephen Todd, David Pearson, Cyril Bourgenot, \\ David Brooks, Robert Snell, Wenjuan Sun, Peter Cooper, \\ Simon G. Alcock and loana-Theodora Nistea
}

\section{Published version information:}

Citation: C Atkins et al. "Lightweighting design optimisation for additively manufactured mirrors." Proceedings of SPIE, vol. 11116 (2019): 1111617. Is in: Astronomical Optics: Design, Manufacture, and Test of Space and Ground Systems II, proceedings of SPIE Optical Engineering + Applications, San Diego, California, USA, 11-15 Aug 2019.

DOI: $\underline{10.1117 / 12.2528105}$

(C) 2019 Society of Photo Optical Instrumentation Engineers (SPIE). One print or electronic copy may be made for personal use only. Systematic reproduction and distribution, duplication of any material in this publication for a fee or for commercial purposes, or modification of the contents of the publication are prohibited.

This version is made available in accordance with publisher policies. Please cite only the published version using the reference above. This is the citation assigned by the publisher at the time of issuing the APV. Please check the publisher's website for any updates. 


\section{Lightweighting design optimisation for additively manufactured mirrors}

Carolyn Atkins, William Brzozowski, Naomi Dobson, Maria Milanova, Stephen Todd, et al.

Carolyn Atkins, William Brzozowski, Naomi Dobson, Maria Milanova, Stephen Todd, David Pearson, Cyril Bourgenot, David Brooks, Robert Snell, Wenjuan Sun, Peter Cooper, Simon G. Alcock, loana-Theodora Nistea, "Lightweighting design optimisation for additively manufactured mirrors," Proc. SPIE 11116, Astronomical Optics: Design, Manufacture, and Test of Space and Ground Systems II, 1111617 (9 September 2019); doi: 10.1117/12.2528105

Event: SPIE Optical Engineering + Applications, 2019, San Diego, California, United States 


\title{
Lightweighting design optimisation for additively manufactured mirrors
}

\author{
Carolyn Atkins*a, William Brzozowski ${ }^{\mathrm{a}}$, Naomi Dobson ${ }^{\mathrm{a}}$, Maria Milanova ${ }^{\mathrm{a}}$, Stephen Todd ${ }^{\mathrm{a}}$, \\ David Pearson ${ }^{\mathrm{b}}$, Cyril Bourgenot ${ }^{\mathrm{c}}$, David Brooks ${ }^{\mathrm{d}}$, Robert Snelle, Wenjuan Sun ${ }^{\mathrm{f}}$, Peter \\ Cooper ${ }^{\mathrm{f}}$, Simon G. Alcock ${ }^{\mathrm{g}}$, and Ioana-Theodora Nistea ${ }^{\mathrm{g}}$ \\ ${ }^{a}$ UK Astronomy Technology Centre, Royal Observatory, Edinburgh, EH9 3HJ, UK \\ ${ }^{\mathrm{b}} \mathrm{RAL}$ Space, Harwell Science \& Innovation Campus, OX11 0QX, UK \\ ${ }^{c}$ Durham University, NETPark Research Institute, Sedgefield, TS21 3FB, UK \\ ${ }^{\mathrm{d} U n i v e r s i t y ~ C o l l e g e ~ L o n d o n, ~ D e p a r t m e n t ~ o f ~ P h y s i c s ~ a n d ~ A s t r o n o m y, ~ L o n d o n, ~ W C 1 E ~ 6 B T, ~ U K ~}$ \\ eDept of Materials Science and Engineering, University of Sheffield, Sheffield, S1 3JD, UK \\ ${ }^{\mathrm{f}}$ National Physical Laboratory, Teddington, Middlesex, TW11 0LW, UK \\ ${ }^{g}$ Diamond Light Source, Harwell Science \& Innovation Campus, OX11 0QX, UK
}

\begin{abstract}
Design for additive manufacture (AM; 3D printing) is significantly different than design for subtractive machining. Although there are some limitations on the designs that can be printed, the increase in the AM design-space removes some of the existing challenges faced by the traditional lightweight mirror designs; for example, sandwich mirrors are just as easy to fabricate as open-back mirrors via AM, and they provide an improvement in structural rigidity. However, the ability to print a sandwich mirror as a single component does come with extra considerations; such as orientation upon the build plate and access to remove any temporary support material.

This paper describes the iterations in optimisation applied to the lightweighting of a small, $84 \mathrm{~mm}$ diameter by $20 \mathrm{~mm}$ height, spherical concave mirror intended for CubeSat applications. The initial design, which was fabricated, is discussed in terms of the internal lightweighting design and the design constraints that were imposed by printing and post-processing. Iterations on the initial design are presented; these include the use of topology optimisation to minimise the total internal strain energy during mirror polishing and the use of lattices combined with thickness variation i.e. having a thicker lattice in strategic support locations. To assess the suitability of each design, finite element analysis is presented to quantify the print-through of the lightweighting upon the optical surface for a given mass reduction.
\end{abstract}

Keywords: Finite element analysis, topology optimisation, additive manufacturing, 3D printing, lightweight mirrors, mirror fabrication

\section{INTRODUCTION}

One of the primary benefits of additive manufacture (AM; 3D printing) is the freedom of design that is available. The layer-upon-layer production method, which is additive as opposed to subtractive, promotes designs that could not have been fabricated via traditional manufacturing methods. The advantages for mirror development are in the broad range of lightweighting structures that can be implemented and the ability to consolidate parts - i.e. to integrate a mirror and mount within single part. The freedom of design places emphasis on optimising for function, rather than for manufacture, which, in the case of mirrors, could lead a reduction in surface form error for a given mass reduction.

AM is increasingly being trialled in metal mirror manufacture with encouraging results ${ }^{1-4}$ and, in addition, the benefit and scope of optimising mirror lightweighting has been discussed by a number of groups. ${ }^{5-8}$ Traditionally, mirror lightweighting techniques have either involved the removal of material from the back of the mirror (open back mirror), or have required a composite structure with a lightweight mesh or lattice placed between two

\footnotetext{
*E-mail: carolyn.atkins@stfc.ac.uk
} 
face plates (sandwich mirror). ${ }^{9}$ Most often, the open back mirrors are easier to fabricate than their sandwich mirror counterparts, but with an open structure at the back they are less rigid and more prone to distortion. ${ }^{10}$ Potentially this compromise in mirror performance can be counteracted using AM, where a sandwich mirror can be printed directly, rather than constructed as a composite. In addition, regular pocketed mass removal patterns used in open-back mirrors can be optimised for the anticipated loads or mounting fixtures, the resultant optimised pockets would potentially be irregular, posing a challenge for traditional machining methods (mill, drill and lathe).

However, the creation of an intricate lightweight mirror core, or applying part consolidation can pose a challenge when generating the optical surface, or machining the areas of interface. AM methods, particularly powder bed fusion which is commonly used for metal AM, print structures with a rough surface finish, Roulet et al. $2018^{11}$ measured approx. $130 \mu \mathrm{m}$ peak to valley (PV) and $16 \mu \mathrm{m}$ root mean square (RMS) over a $38 \mathrm{~mm}$ Form Talysurf line profile on a raw aluminium alloy print. Therefore, in the majority of cases, traditional machining techniques will be required on an AM mirror substrate and as a result consideration must be given to the eventual post-processing procedures to ensure that the AM substrate can be turned into an AM mirror.

This paper describes the design and analysis of an AM mirror specified for use as a primary mirror in a $3 \mathrm{U}$ CubeSat telescope for Earth observation. The initial design, which was fabricated to investigate different AM materials and post-processing methods (diamond turning versus polishing), ${ }^{12}$ is presented in Section 2 and includes a description of how the AM design incorporated restrictions imposed by AM manufacture and traditional machining. Section 3 describes different approaches in which the AM mirror design can be optimised to improve functionality - i.e. to increase mass reduction and reduce optical surface form errors.

\section{MIRROR DESIGN}

\subsection{CubeSat Camera: mirror design}

The dimensions of the fabricated AM mirror were defined by an existing telescope design specified within the CubeSat Camera $(\mathrm{CCAM})^{13}$ paper study. The CubeSat configuration for this study was $3 \mathrm{U}(100 \mathrm{~mm} \times 100 \mathrm{~mm}$ $\times 300 \mathrm{~mm}$ ) where the telescope system occupies $1.2 \mathrm{U}$. The telescope is a Cassegrain configuration with a total focal length of $440 \mathrm{~mm}$ packaged within the $1.2 \mathrm{U}$; the primary mirror (M1) is $84 \mathrm{~mm}$ diameter with a $2 \mathrm{~mm}$ flat bevel on the circumference; and a $32 \mathrm{~mm}$ diameter hole is located in the centre of the mirror. The maximum height of the M1 mirror is approx. $12 \mathrm{~mm}$ and was designed to be a solid mirror fabricated using the glass BK7.

\subsection{Mirror design requirements}

The adapted requirements for the AM mirror variant are presented in Table 1 and the initial concept drawing is shown in Figure 1. The parameter, substrate thickness, within Table 1 is the thickness of the external skin shown in Figure 1. The internal cavity, shown in blue within the figure, highlights the volume which is not constrained by design requirements and is open to AM design - including lightweight structures and mounting fixtures. The optical prescription was simplified to a spherical concave approximation of the CCAM M1 optical design; this ensured a simple metrology set-up to quantify the surface form error of the fabricated mirrors. ${ }^{12}$

Table 1. AM mirror design requirements

\begin{tabular}{|l|l|}
\hline Property & Value \\
\hline Optical prescription & Spherical, concave \\
Radius of curvature & $350 \mathrm{~mm}$ \\
Clear aperture diameter & $80 \mathrm{~mm} \varnothing$ \\
Mechanical diameter & $84 \mathrm{~mm} \varnothing$ \\
Substrate thickness (skin) & $2 \mathrm{~mm}$ \\
Total height (inclu. mount) & $17.3 \mathrm{~mm}$ \\
\hline
\end{tabular}




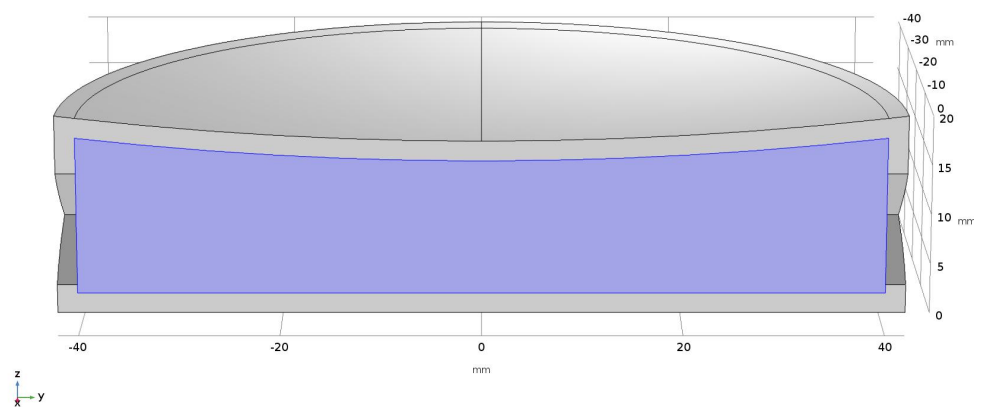

Figure 1. A cross-section of the first iteration on the AM mirror design which incorporates the requirements described in Table 1. The internal cavity (blue) is the region to be lightweighted and to include the mounting fixtures; the external grey skin is defined by the design requirements and is geometrically fixed.

\subsection{AM design considerations}

In addition to the mirror specifications listed in Table 1, there were further design criteria influenced by AM build preferences and post-processing considerations, as listed below.

- Lattice - an internal lattice was desired to demonstrate a structure that would not have been possible via traditional machining.

- Print orientation - a vertical print orientation was desired to ensure that the mirror surface would be self-supporting and facilitate the removal of excess support material.

- Powder removal - holes to remove unsolidified powder between the lattice are required, it was desired that these holes were not located on the mirror wall in order to aid machining.

- Post-processing - to generate the mirror surface and to allow the mirror to be integrated within mounting hardware, the printed design should interface with ease to traditional machining equipment.

\subsubsection{Lattice choice}

A lattice was desired in order to trial a structure that was only possible via AM. There are a large variety of lattice options available, especially if commercial AM design software is used; however, due to time and software limitations, the lattice selection was restricted to: body centred cubic (BCC), body centred cubic with z axis reinforcement $(\mathrm{BCCz})$, rhombic dodecahedron, and truncated octahedron. The four different lattice styles are shown in Figure 2.

Initial finite element analysis (FEA) suggested that $\mathrm{BCC}$ and $\mathrm{BCCz}$ lattices would provide the optimum support of the mirror surface to negate the effects of mirror fabrication print-through (i.e. the quilting effect; discussed in Section 3); the BCCz lattice, with the additional z-axis support normal the mirror surface, provided the best performance.

\subsubsection{Print orientation}

Print orientation refers to the orientation of a part relative to the build platform ( $x$ - $y$ plane) of the AM machine. How a part is orientated affects the structures that can be built without the requirement of excess support material. Typically, if the angle of the surface to the build platform is between $45^{\circ} \longrightarrow 90^{\circ}$ then the surface is self-supporting and does not require excess support material. Reducing the support material in a build is desirable from a cost-time consideration, for example, if support material is required to support an internal lattice structure during build, then time will have to be spent removing this enclosed waste material after the 


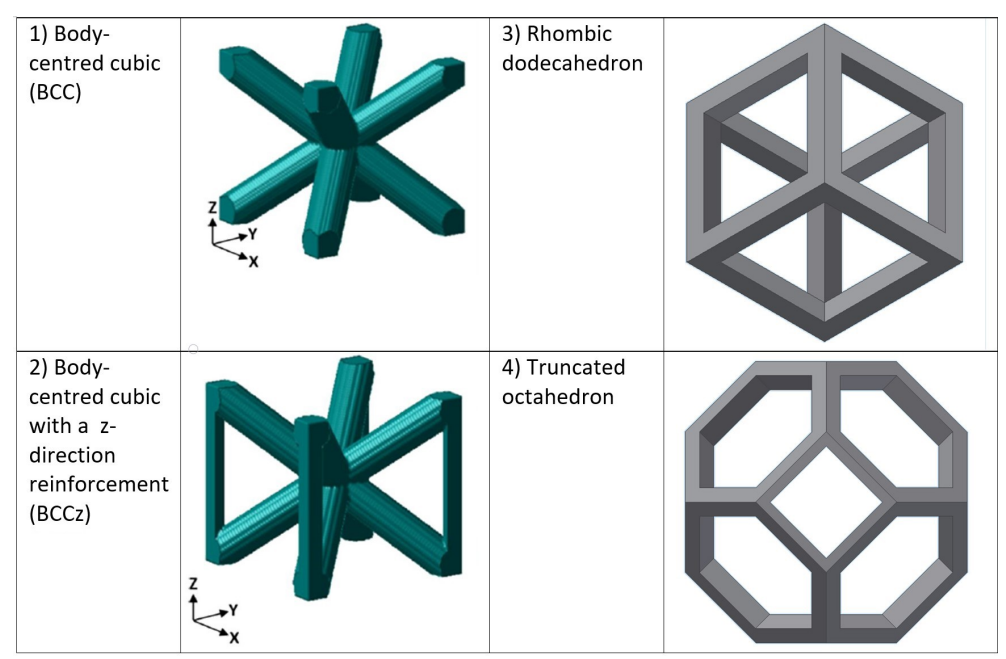

Figure 2. The four lattice variants that were investigated. The BCC and BCCz images are credited to Panesar et al. 2018. ${ }^{14}$

build. However, it should be noted that support material on the external faces of a part plays an important role in heat dissipation during the build. ${ }^{15}$ Figure 3 highlights a cross-section of the mirror structure and the anticipated internal and external supports required to build the part.

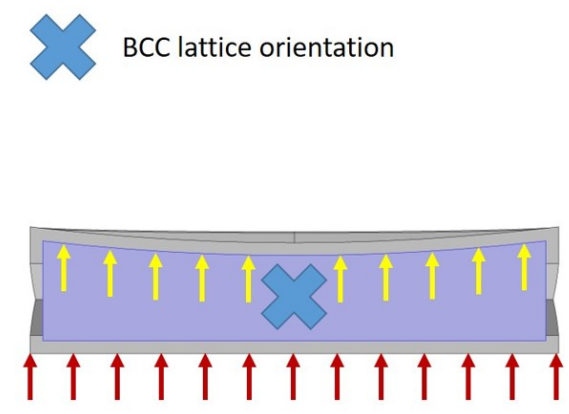

a)

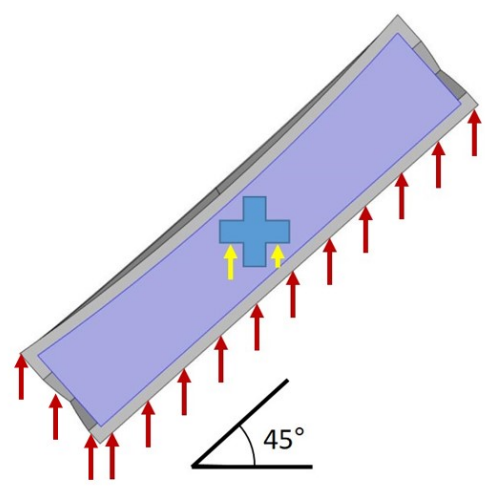

b)

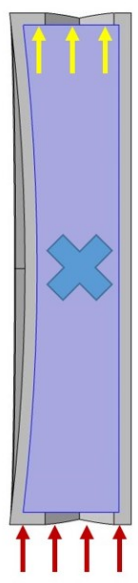

c)

Figure 3. The print orientation of the mirror and the effect on internal and external support material: a) $0^{\circ}$ to the build platform requires support along the base and internally to support the mirror surface; b) $45^{\circ}$ to the build platform requires external support material for heat dissipation and internal support for the lattice; and c) $90^{\circ}$ to the build platform, external support material required on the base and internal supports required on the side wall.

A further consideration regarding the build orientation, is how it affects the performance of the mirror surface. Atkins et al. $2018^{16}$ presented a series of surface roughness measurements that depicted observable print layers on the optical surface of an AM aluminium mirror printed in the vertical orientation. In comparison to a cast block of aluminium, these layers do contribute to surface roughness and therefore, arguably, alternative orientations could provide improved results; further investigation into the effect of build orientation and surface roughness is on-going. Within this study, to mitigate the risk of printing in an unknown orientation, the decision was made to print vertically as the previous results were within the desired optical performance outlined within 
Atkins et al. 2019. ${ }^{12}$

The decision to print in the vertical orientation influenced the lattice selection. The favoured $\mathrm{BCCz}$ lattice, with the additional struts normal to the optical surface, could not be printed in a vertical orientation without internal support material, likewise a BCC lattice printed at $45^{\circ}$ would incur a similar challenge - as demonstrated in Figure $3 \mathrm{~b}$ ). Therefore printing vertically, with a BCC lattice, minimises the use of both internal and external support material.

\subsubsection{Powder removal}

The internal cavity, populated with the BCC lattice, required access holes to the external faces to allow removal of excess powder from the build. Within the design space, the access holes could be situated either on the base, or on the side wall. An important requirement when designing a part for AM is to ensure that the part can be machined on the function critical surfaces. During the mirror design phase, it was decided to machine all the external faces of the mirror and therefore, to facilitate this requirement, the access holes were placed upon the base of the mirror.

\subsubsection{Post-processing}

To aid the machining of the AM mirror, a sacrificial spigot was situated upon the base of the mirror. The spigot would be used to hold of the AM substrate within the spindle of the lathe and then removed when the machining was complete.

\subsection{The final design}

The final design incorporated the design requirements as outlined in Section 2.3 and is presented in Figure 4. To allow the design to be printed in the vertical orientation without the need for internal support material, additional material was added to the corners of the internal side walls to create a $45^{\circ}$ build angle, resulting in an ellipsoidal cavity - as shown in Figure 4c).

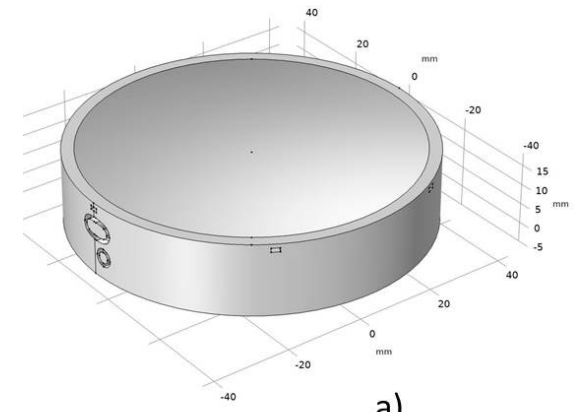

a)

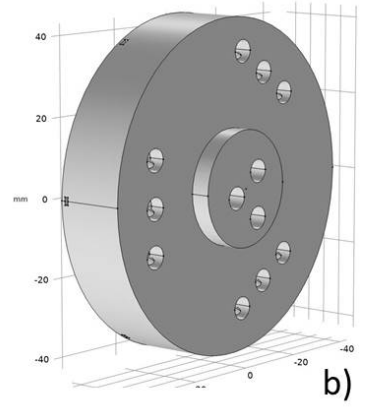

b)

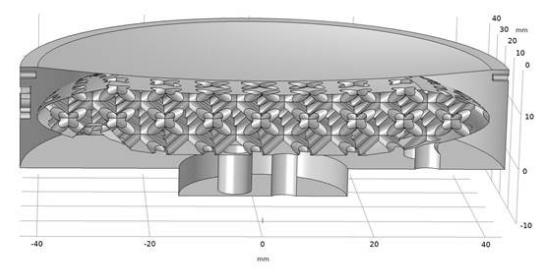

c)

Figure 4. A CAD representation of the AM M1 mirror to print: a) external mirror surface with fiducials; b) the base of the mirror highlighting the sacrificial spigot and powder removal holes; and c) the internal structure of the mirror.

The lightweighting of the mirror design was relatively modest; the design has $\sim 69 \%$ the weight of a solid equivalent (i.e. $\sim 31 \%$ of the mass has been removed). The project requirement was to compare the mirror quality that could be achieved via diamond turning and polishing upon AM substrates. Therefore the design of the mirror substrate needed to be low risk in terms of structural rigidity to ensure a good optical surface, while simultaneously demonstrating some of the unique geometries available through AM. Parallel to the postprocessing of the AM substrates, design optimisation of the AM mirror design was undertaken to investigate alternative design approaches that are available through AM; Section 3 describes these efforts. 


\section{DESIGN OPTIMISATION}

The primary advantage in the use of additive manufacture in the majority of product design cases is the breadth of structural choice. In mirror fabrication this is particularly beneficial to create lightweight, rigid structures to reduce the mirror mass while maintaining the desired optical profile. This study sampled a few of the innovative techniques and structures that can now be implemented using AM: topology optimisation (TO), lattice generation, and structures from nature. To assess the different approaches, the designs are compared against an FEA of the manufactured design detailing the surface distortion and the associated percentage of lightweighting.

\subsection{Finite element analysis}

To create a benchmark by which the suitability of the alternative structures can be assessed, the original design, as outlined in Section 2, was evaluated for the polishing print-through effect, commonly known as quilting. This effect occurs due to the uneven support of the mirror substrate by the lightweighting structure beneath and results in a physical imprint of the lightweighting cavities upon the mirror surface - illustrated in Figure 5.

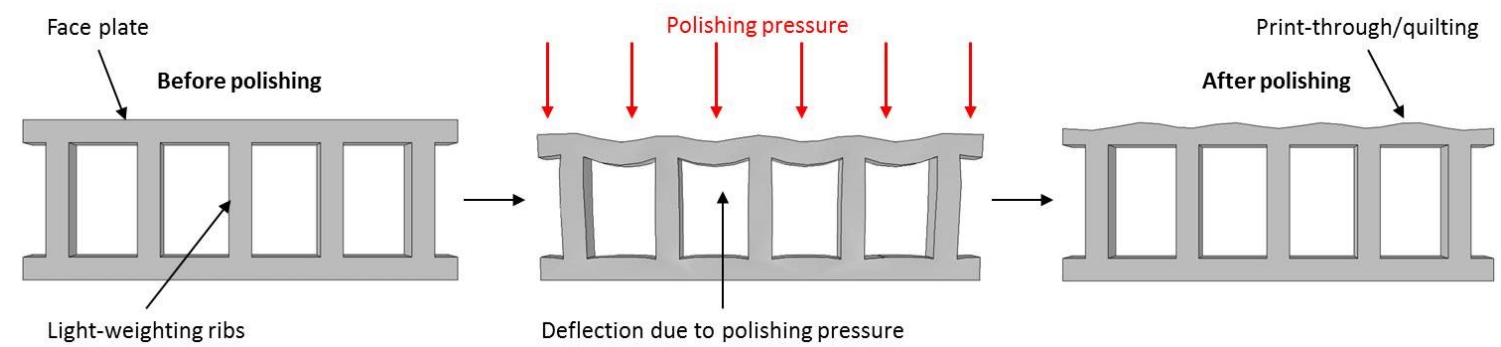

Figure 5. An illustration of the surface distortions created during the polishing process of a lightweighted structure.

\subsubsection{Model set up}

To simulate the effect of the polishing print-through upon the surface of the mirror, a computer aided design (CAD) representation of the machined AM print was used. COMSOL Multiphysics modelling software was used to perform the FEA and within the model the base of the substrate was fixed in $z$ (i.e. the normal to the face of the base) and edges along the side of the substrate fixed in either $x$ or $y$ as required to constrain the model against twisting. A uniform boundary load of $3500 \mathrm{~N} / \mathrm{m}^{2}$ was used to approximate the pressure applied during polishing - the exact pressure applied to the AM substrates during polish is unknown; however $3500 \mathrm{~N} / \mathrm{m}^{2}$ acts as a standard value for this optimisation study. Figure 6 a) demonstrates the application of the FEA boundary conditions.

\subsubsection{Simulation results and benchmark values}

Figure $6 \mathrm{~b}$ ) presents the z-displacement anticipated from the FEA simulation. The simulation predicts a distortion PV of $10.5 \mathrm{~nm}$ and a root mean square (RMS) of $3.2 \mathrm{~nm}$. Mid order spatial frequencies are visible within the displacement data and these are related to the lattice structure beneath; however, for the creation of an initial benchmark model only the low spatial frequency form error is considered.

Table 2 highlights the three metrics which are used to assess the suitability of the different optimised designs: the percentage of lightweighting (mass remaining); the PV of the z-displacement at $3500 \mathrm{~N} / \mathrm{m}^{2}$; and the RMS of the sag distortion at $3500 \mathrm{~N} / \mathrm{m}^{2}$. By assessing via these criteria the optimum design(s) would be one that exhibits a significant reduction in mass and low values of distortion PV and RMS.

Table 2. Benchmark AM mirror design

\begin{tabular}{|l|l|l|l|}
\hline Mirror & Mass remaining \% & Distortion PV & Distortion RMS \\
\hline Benchmark design & 68.7 & $10.5 \mathrm{~nm}$ & $3.2 \mathrm{~nm}$ \\
\hline
\end{tabular}



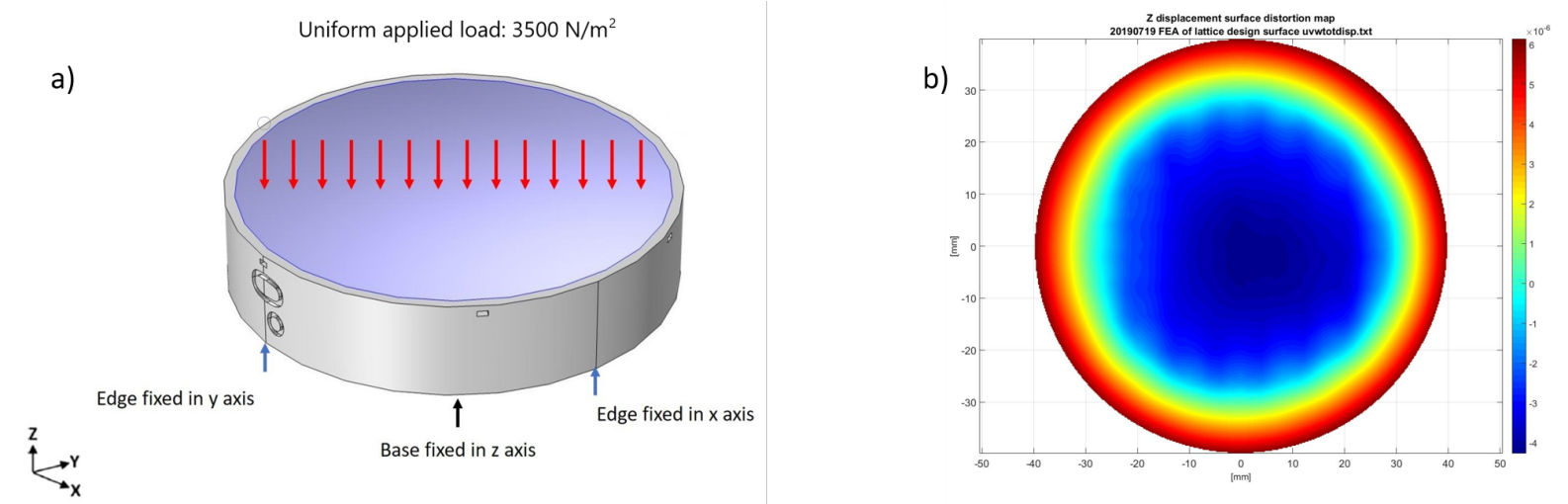

Figure 6. FEA model and simulation result: left the model set-up indicating the applied boundary condition and polishing load; and right the surface deformation in the $\mathrm{z}$ orientation on the mirror surface.

\subsection{Optimisation routes}

The selection of the optimisation routes for this project was dictated by the software available for use within the institutions. COMSOL Multiphysics, in conjunction with the Optimisation and Structural Mechanics modules, allowed for topology optimisation (TO) and the creation of regular 'structures from nature'. Through collaboration with the University of Sheffield, the dedicated AM software NetFabb was implemented to trial different lattice structures, which could be generated automatically rather than drawn manually within CAD. This optimisation study therefore highlights a selection of alternative approaches that can be applied to mirror lightweighting, but these do not constitute all the possible structures for study.

\subsection{Topology optimisation}

Topology optimisation allows a designer to optimise a structure within a volume for a given set of boundary conditions and a defined volume fraction (vol. frac.). The volume fraction is the fraction of the volume that is desired after optimisation - e.g. 0.4 refers to $40 \%$ of the mass remaining after optimisation. In this study TO is applied by replicating the FEA model described Section 3.1 and then iterating in order to minimise the total internal strain energy of the system while limited by the defined volume fraction. A further description of the process can be found in Atkins et al. $2018 .{ }^{16}$

Figure 7 depicts the internal volume for optimisation and the external volume representing the mirror design requirement and mounting supports; two variants were simulated, one with and one without a side wall. COMSOL Multiphysics version 5.4 was used for the optimisation; the example for a loaded beam ${ }^{17}$ was followed to apply the same method to the this study. Two series of iterations were performed to output the final design, the first optimisation used a coarse mesh to obtain the approximate structure and then a second optimisation used a finer mesh to enhance the solution.

\subsubsection{Topology optimised structures}

Figure 8 highlights the optimised structures in the $x-y$ plane from the mirror surface. In each solution, the optimised structure incorporate the solid mounting cones. In all except Vol. frac. 0.3, no side walls, a annular structure for the solution is observed. This result is to be expected considering the geometry of the mirror and a similar solution was obtained in the study by Atkins et al. 2017. ${ }^{18}$ The difference between the no side walls and with side walls variants, is that in the latter the annular structure has a smaller annular radius of curvature, as the edge of the mirror is already supported; whereas the former must support the entirety of the underside of the mirror and therefore the annular radius of curvature is larger. 


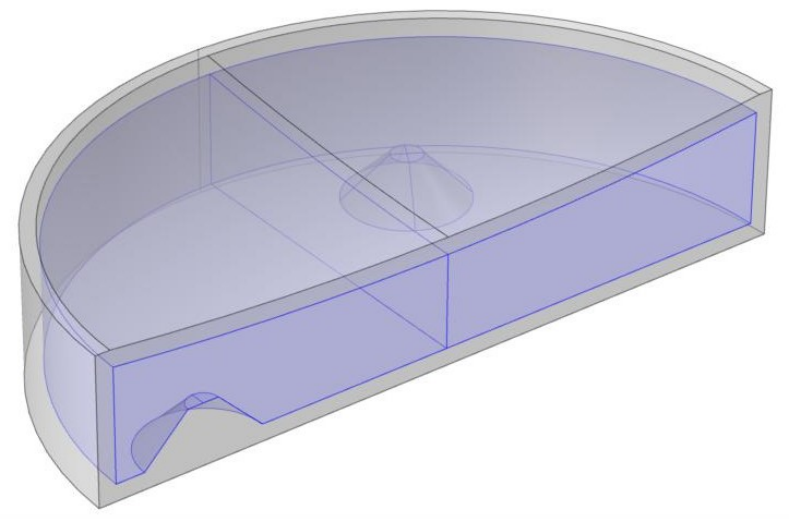

Figure 7. A cross section depicting half of the mirror substrate used in the optimisation. The blue highlighted core is the volume for optimisation, whereas the outer shell and the mounting points remain.

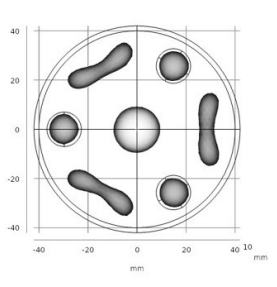

Vol. frac. 0.3

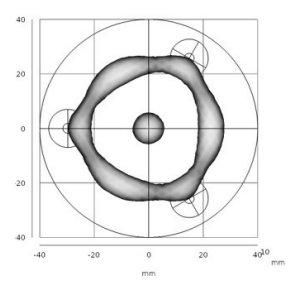

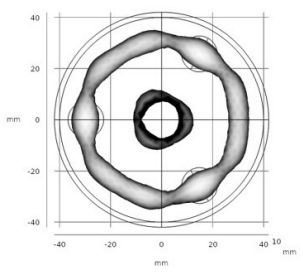

Vol. frac. 0.4

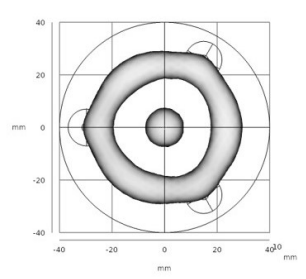

No side walls

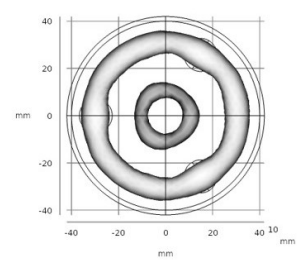

Vol. frac. 0.5
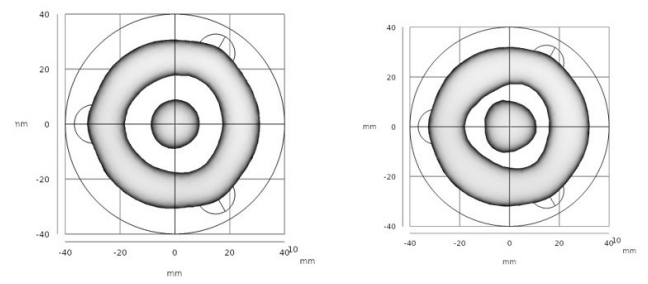

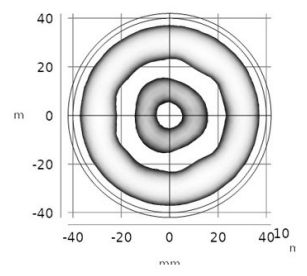

Vol. frac. 0.7

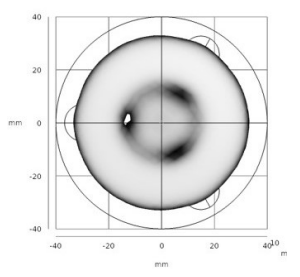

\section{With side walls}

Figure 8. The optimised geometries (shaded in grey) as seen above the optical surface in the $x$ - $y$ plane. The upper five plots were simulated with no side walls and the lower five plots with a $2 \mathrm{~mm}$ side wall. 


\subsubsection{Analysis of topology optimised solutions}

To assess the suitability of the optimised solutions, the percentage of lightweighting (i.e. the mass remaining) and the PV and RMS of the $\mathrm{z}$ displacement data was calculated for the applied $3500 \mathrm{~N} / \mathrm{m}^{2}$ vertical load. In the calculation of the mass remaining percentage Equation 1 was used, where $V_{\text {shell }}$ is the non-optimised volume, $V_{\text {core }}$ is the volume to optimise, $V_{\text {total }}=V_{\text {shell }}+V_{\text {core }}$, and vol. frac. is the fraction of the volume remaining after optimisation. The volumes were generated within the COMSOL Multiphysics Geometry environment.

$$
\text { Mass remaining } \%=\frac{V_{\text {shell }}}{V_{\text {total }}}+\frac{\text { Vol. frac. } \times V_{\text {core }}}{V_{\text {total }}}
$$

Table 3 collates the results obtained from the surface displacement maps derived from the topologies in Figure 8 and the magnitude of the lightweighting achieved. Predictably, the results demonstrate that the maximum distortions are observed with the greatest reduction in mass, but the presence of a side wall does not guarantee a reduction in distortion. The addition of the side walls increased the simulation constraints and limits the optimal mass distribution that can be obtained per model.

Comparing the TO results with those presented in the FEA benchmark (Table 2), there is a reduction in surface form error for the equivalent percentage of mass reduction, which highlights the potential of TO to redesign mirror lightweighting.

Table 3. Surface displacement and mass reduction $\%$ for the TO simulations.

\begin{tabular}{|l|l|l|l|}
\hline Design, Vol. frac. & Mass remaining \% & distortion PV & distortion RMS \\
\hline No walls, 0.3 & 48.1 & $20 \mathrm{~nm}$ & $4.0 \mathrm{~nm}$ \\
No walls, 0.4 & 55.5 & $9.2 \mathrm{~nm}$ & $2.0 \mathrm{~nm}$ \\
No walls, 0.5 & 62.9 & $4.2 \mathrm{~nm}$ & $1.0 \mathrm{~nm}$ \\
No walls, 0.6 & 70.3 & $2.6 \mathrm{~nm}$ & $0.59 \mathrm{~nm}$ \\
No walls, 0.7 & 77.8 & $1.9 \mathrm{~nm}$ & $0.41 \mathrm{~nm}$ \\
\hline \hline Side walls, 0.3 & 53.4 & $7.8 \mathrm{~nm}$ & $2.2 \mathrm{~nm}$ \\
Side walls, 0.4 & 60.1 & $5.1 \mathrm{~nm}$ & $1.5 \mathrm{~nm}$ \\
Side walls, 0.5 & 66.7 & $4.0 \mathrm{~nm}$ & $1.1 \mathrm{~nm}$ \\
Side walls, 0.6 & 73.4 & $2.7 \mathrm{~nm}$ & $0.81 \mathrm{~nm}$ \\
Side walls, 0.7 & 80.0 & $2.1 \mathrm{~nm}$ & $0.68 \mathrm{~nm}$ \\
\hline
\end{tabular}

\subsection{Lattice generation}

Commercial software for AM design have the capability to generate a variety of lattice structures in a given volume - as opposed to the benchmark design, where the lattice was generated in CAD using a replicated single cell. In collaboration with the University of Sheffield, the lattices available within the NetFabb Ultimate ${ }^{19}$ were evaluated for their suitability as mirror lightweighting. NetFabb has 19 different lattice geometries available to the designer and these can be optimised in terms of strut thickness to provide improved rigidity.

\subsubsection{Evaluation of lattice options}

All the lattices within NetFabb were evaluated to quantify their suitability as mirror lightweighting. To perform this analysis, a cylinder with the approximate dimensions of the benchmark mirror ( $42 \mathrm{~mm}$ radius and $16 \mathrm{~mm}$ in height) was used as the volume to populate the lattice. Each lattice cell was defined within a cube with a $5 \mathrm{~mm}$ length and the thickness of the struts defining the lattice structure were $0.5 \mathrm{~mm}$. Two cylindrical face plates with a thickness of $2 \mathrm{~mm}$ were added to sandwich the lattice volume - as shown in Figure 9. For each lattice, a force of $3500 \mathrm{~N} / \mathrm{m}^{2}$ was applied normal to the mirror surface and then applied parallel to the mirror surface; the maximum displacement was recorded for each case. In addition, the mass remaining $\%$ of the mirror structure 


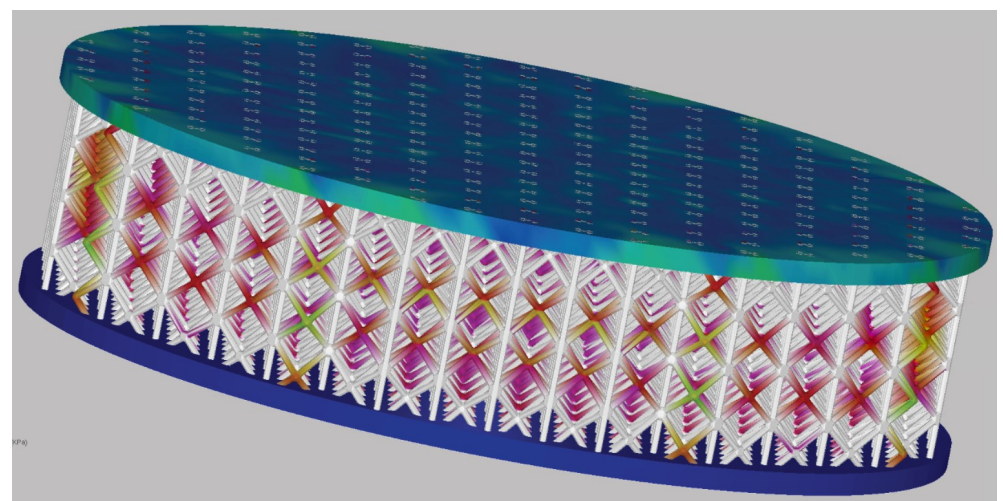

Figure 9. A representation of how the Star lattice has been used to populate the lightweighting volume.

was recorded to evaluate the magnitude of the mass reduction. Table 4 highlights the assessment of each of the lattices; unfortunately a result could not be obtained for the 3D Spider lattice.

The goal of the first evaluation was to down select to three lattice structures for further study. Each of the numerical columns within Table 4 were ranked from 1 (lowest mass remaining/ minimum displacement) to 19 (highest mass remaining or maximum displacement). A weighted mean was then used to rank the lattices; the displacements caused by the normal and parallel loads were given twice the weighting than the mass remaining $\%$, as the priority was to generate a rigid structure. It should be noted that the force was only applied in one parallel direction and due to the geometry of some of the lattice cells, an anisotropic behaviour in displacement is expected. Following the weighted mean, the down selected lattice structures were: Star $\left(1^{\text {st }}\right)$, Icosahedron $\left(2^{\text {nd }}\right)$ and Tetra $\left(3^{r d}\right)$; shown in Figure 10.

\subsubsection{Evaluation of unit cell size}

Following the down selection, the three unit cells were evaluated in terms of the length of the cubic unit cell. The strut thickness of the lattice was constant at $0.5 \mathrm{~mm}$, but the unit cell length was increased from $2 \mathrm{~mm}$ to a maximum of $16 \mathrm{~mm}$, where 512 of the $2 \mathrm{~mm}$ cell size fit within the $16 \mathrm{~mm}$ length volume.

The two plots in Figure 11 highlight the change in maximum displacement as a function of cell size length and mass remaining percentage for the three down selected lattices. The upper plot demonstrates the change in displacement with cubic cell length: the star lattice exhibits the minimum change in displacement with cell length although there is obvious scatter in the recorded displacement between the odd and even cell size values above $10 \mathrm{~mm}$; and the tetra and icosahedron lattices demonstrate a systematic increase in displacement with cell length, with the tetra lattice demonstrating the highest magnitude in displacements. The lower graph, replots the cell size as a function of mass remaining percentage: the star lattice presents a clear reduction in weight for given displacement in relation to the other lattices; and the icosahedron lattice when taken in context with weight no longer provides an improvement in displacement relative to the tetra lattice - the denser icosahedron lattice structure is shown in Figure 10. Both plots indicate that the star lattice provides the optimum performance, despite the variability observed above $10 \mathrm{~mm}$, this is expected due to the vertical struts within the lattice that directly oppose the applied load as shown in the $x$ - $z$ plane subplot in Figure 10.

\subsubsection{Lattice optimisation}

The previous section identified the Star lattice as offering a lower maximum displacement for a given mass fraction and therefore selected for optimisation within NetFabb. First, a model was created following the benchmark design in Section 3.1, internally the stress within the lattice is compared against a user set threshold value, if the stress exceeds the threshold value then additional material is added to the lattice struts, however, if the threshold value is not exceeded then the optimisation exits (no material is removed from the struts). To create a structure that does not fracture under a load, the threshold stress value should be lower the yield strength of the material. 
Star lattice (BCCZ)

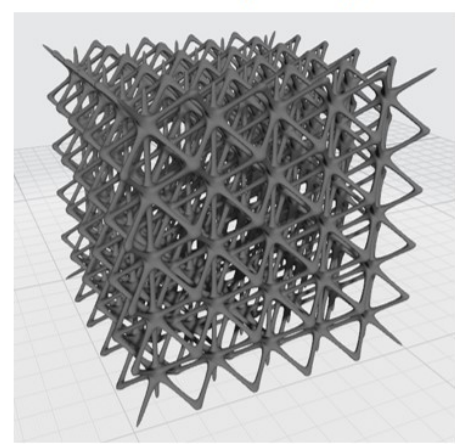

$(x, y, z)$

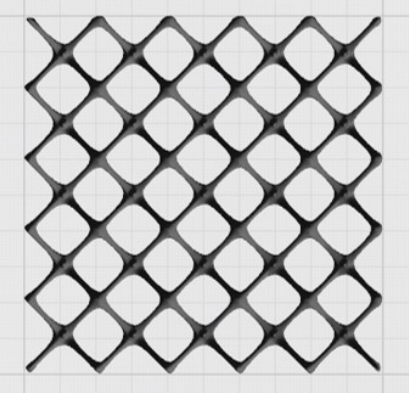

$(x, y)$

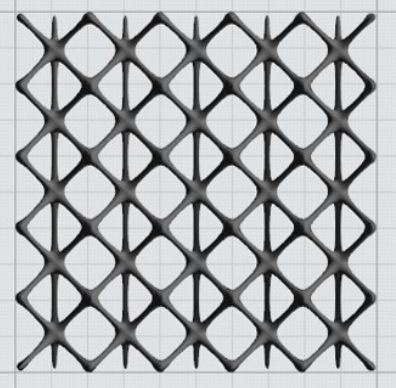

$(x, z)$

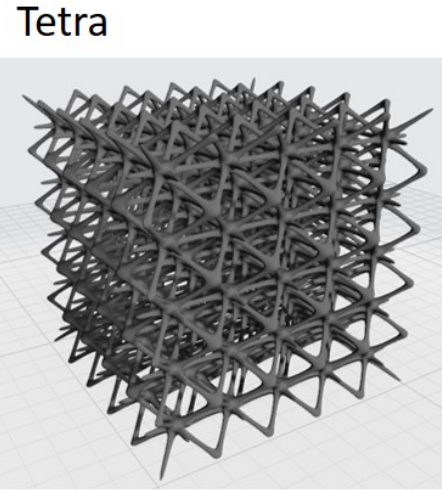

$(x, y, z)$

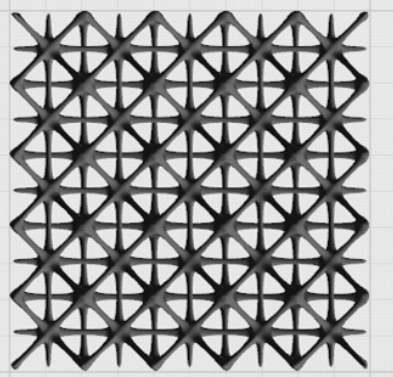

$(x, y)$

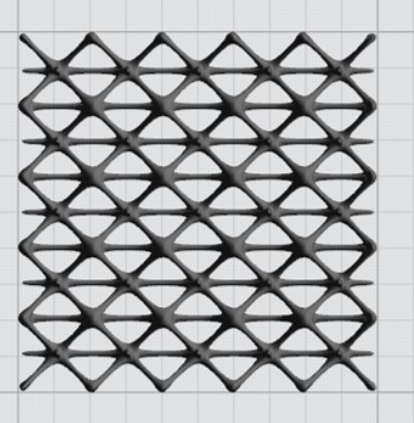

$(x, z)$

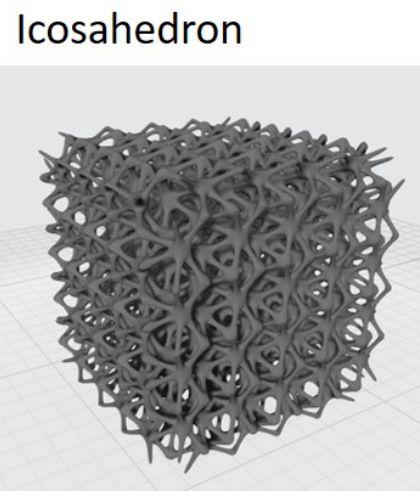

$(x, y, z)$

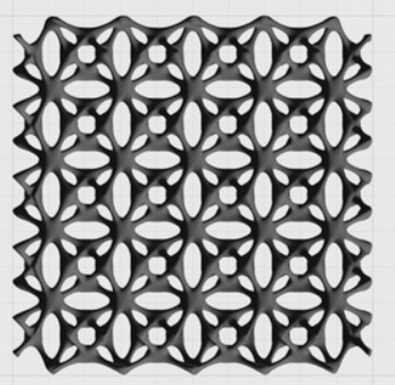

$(x, y)$

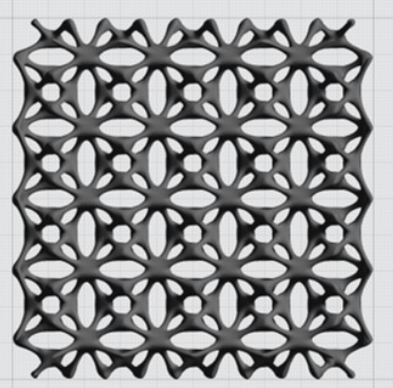

$(x, z)$

Figure 10. Graphical representations of the three selected lattices: top - Star lattice which is equivalent to the BCCz lattice arrangement described in Figure 2; middle - the Tetra lattice; and bottom the Icosahedron lattice. 
Table 4. Evaluation of lattice geometries for loads normal and parallel to the mirror surface

\begin{tabular}{|l|l|l|l|}
\hline \multirow{2}{*}{ Lattice topology } & \multirow{2}{*}{ Mass remaining $\%$} & \multicolumn{2}{|c|}{ Total displacement [nm] } \\
\cline { 2 - 4 } & & Normal load & Parallel load \\
\hline Grid & 21.8 & $7.4 \times 10^{1}$ & $1.1 \times 10^{4}$ \\
X & 24.7 & $1.5 \times 10^{2}$ & $2.1 \times 10^{2}$ \\
Star & $\mathbf{2 5 . 7}$ & $\mathbf{4 . 9} \times \mathbf{1 0}^{\mathbf{1}}$ & $\mathbf{1 . 2} \times \mathbf{1 0}^{\mathbf{2}}$ \\
W & 25.1 & $1.8 \times 10^{2}$ & $2.5 \times 10^{2}$ \\
Column & 19.8 & $7.4 \times 10^{1}$ & $1.1 \times 10^{5}$ \\
Dark horse & 24.1 & $1.1 \times 10^{2}$ & $7.5 \times 10^{2}$ \\
Vin Tiles & 26.3 & $1.1 \times 10^{2}$ & $5.1 \times 10^{2}$ \\
Cross pattee & 31.2 & $6.9 \times 10^{1}$ & $1.3 \times 10^{3}$ \\
Soft box & 25.6 & $3.4 \times 10^{2}$ & $5.8 \times 10^{2}$ \\
Hexa Grid & 20.7 & $8.5 \times 10^{1}$ & $1.2 \times 10^{4}$ \\
Snow Flake & 28.7 & $1.6 \times 10^{2}$ & $1.3 \times 10^{3}$ \\
Hexagon & 28.5 & $8.9 \times 10^{1}$ & $3.0 \times 10^{2}$ \\
Octagon & 24.3 & $5.4 \times 10^{2}$ & $4.3 \times 10^{3}$ \\
3D spider & 26.9 & - & - \\
Icosahedron & $\mathbf{3 3 . 0}$ & $\mathbf{7 . 4} \times \mathbf{1 0}^{\mathbf{1}}$ & $\mathbf{1 . 3} \times \mathbf{1 0}^{\mathbf{2}}$ \\
Pyritohedron & 28.0 & $1.6 \times 10^{2}$ & $5.2 \times 10^{2}$ \\
Rhombic dodecahedron & 27.0 & $4.8 \times 10^{2}$ & $1.1 \times 10^{3}$ \\
Crush & 33.4 & $3.1 \times 10^{2}$ & $6.6 \times 10^{2}$ \\
Tetra & $\mathbf{2 8 . 1}$ & $\mathbf{1 . 1} \times \mathbf{1 0}^{\mathbf{2}}$ & $\mathbf{1 . 8} \times \mathbf{1 0}^{\mathbf{2}}$ \\
Exoskeleton & 21.7 & $7.5 \times 10^{2}$ & $6.6 \times 10^{3}$ \\
\hline
\end{tabular}

The optimisation of the Star lattice investigated two properties, the length of unit cell and the threshold value of the optimisation. The same unit cell lengths were investigated as described in Section 3.4 .2 and these were combined with threshold stress values of $50 \mathrm{MPa}, 100 \mathrm{MPa}$ and $200 \mathrm{MPa}$. The results of the optimisation are shown in Figure 12; the upper plot directly highlights the effect of unit cell length on maximum displacement and the lower plot depicts the same displacement data against the mass remaining percentage. The upper plot demonstrates a slow increase in displacement with unit cell length up to approx. $10 \mathrm{~mm}$, beyond this value there is increased scatter between the cell sizes in terms of displacement, implying a less stable structure. The lower plot, seems to indicate trend lines similar to those observed in Figure 11; however, when taken in context with the upper plot, the observed 'switch-back' is in fact scatter within the results at the larger cell lengths. These results highlight a first attempt in the use of this method to generate mirror lightweighting and therefore further analysis is required; however, coupling the data from Figures 11 and 12 would suggest an optimum Star lattice cell size less than a $7 \mathrm{~mm}$ cube.

Figure 13 depicts an example of how the software has optimised the Star lattice with a cell length of $8 \mathrm{~mm}$ and a threshold value of $100 \mathrm{MPa}$. The column widths, depicted as circular nodes in the cross-section image, have an increase in thickness relative to the diagonal linking struts; this effect is expected due to the load applied in the normal to the mirror surface. In addition, the outer columns have a greater thickness in comparison to the inner columns due to the lack of uniform support at the edge of the mirror face plate. The lack of uniform support at the edge of the mirror surface is an effect of the lattice geometry and in the case presented in Figure 13, a cubic lattice is fitted to a cylindrical geometry. Therefore, to provide a uniform support for a cylindrical mirror, a lattice with rotational symmetry is required. 

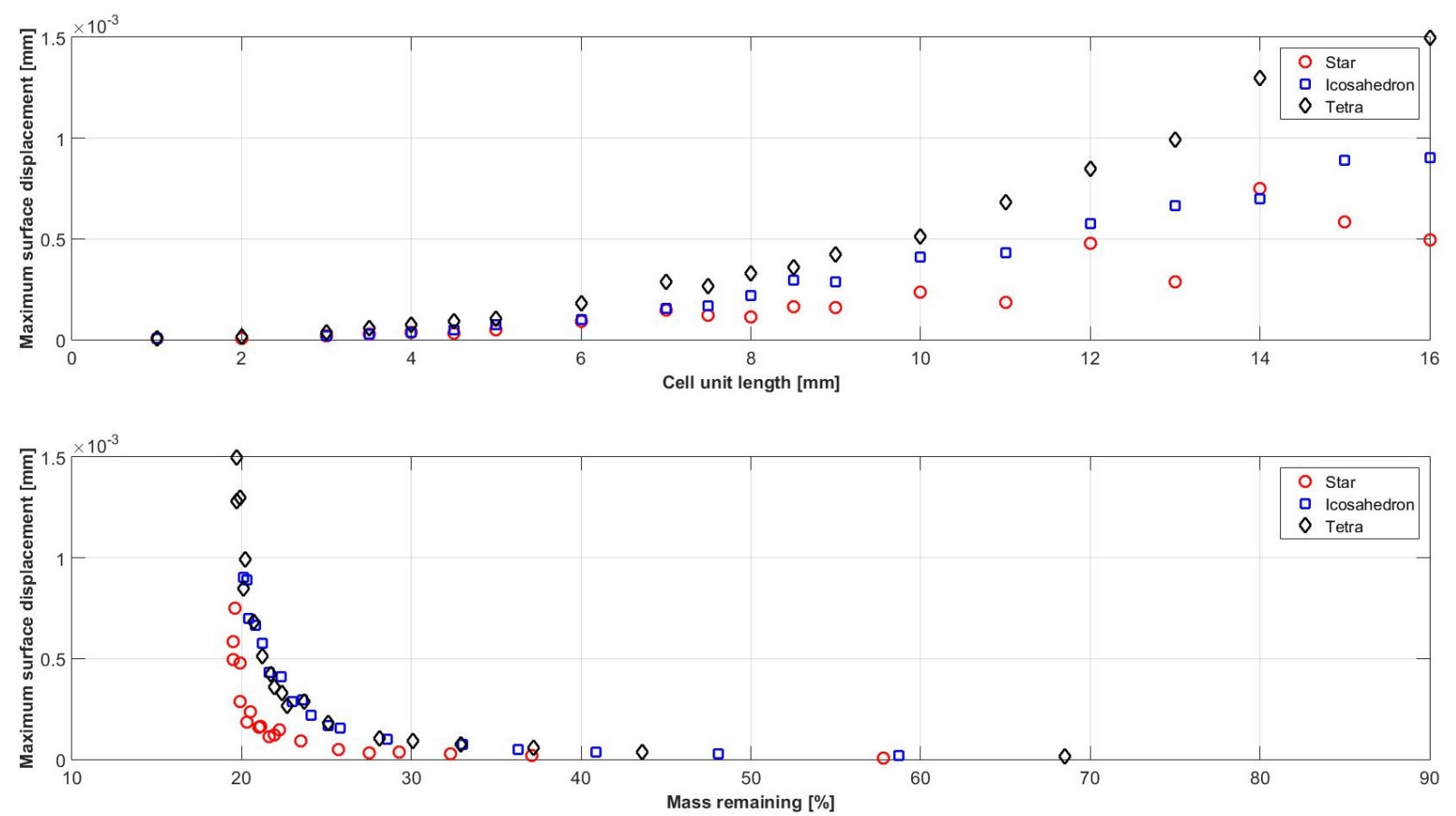

Figure 11. Upper plot - the change in maximum displacement with unit cell length for the three lattice geometries; lower plot - the change in maximum displacement as a function of mass remaining percentage.
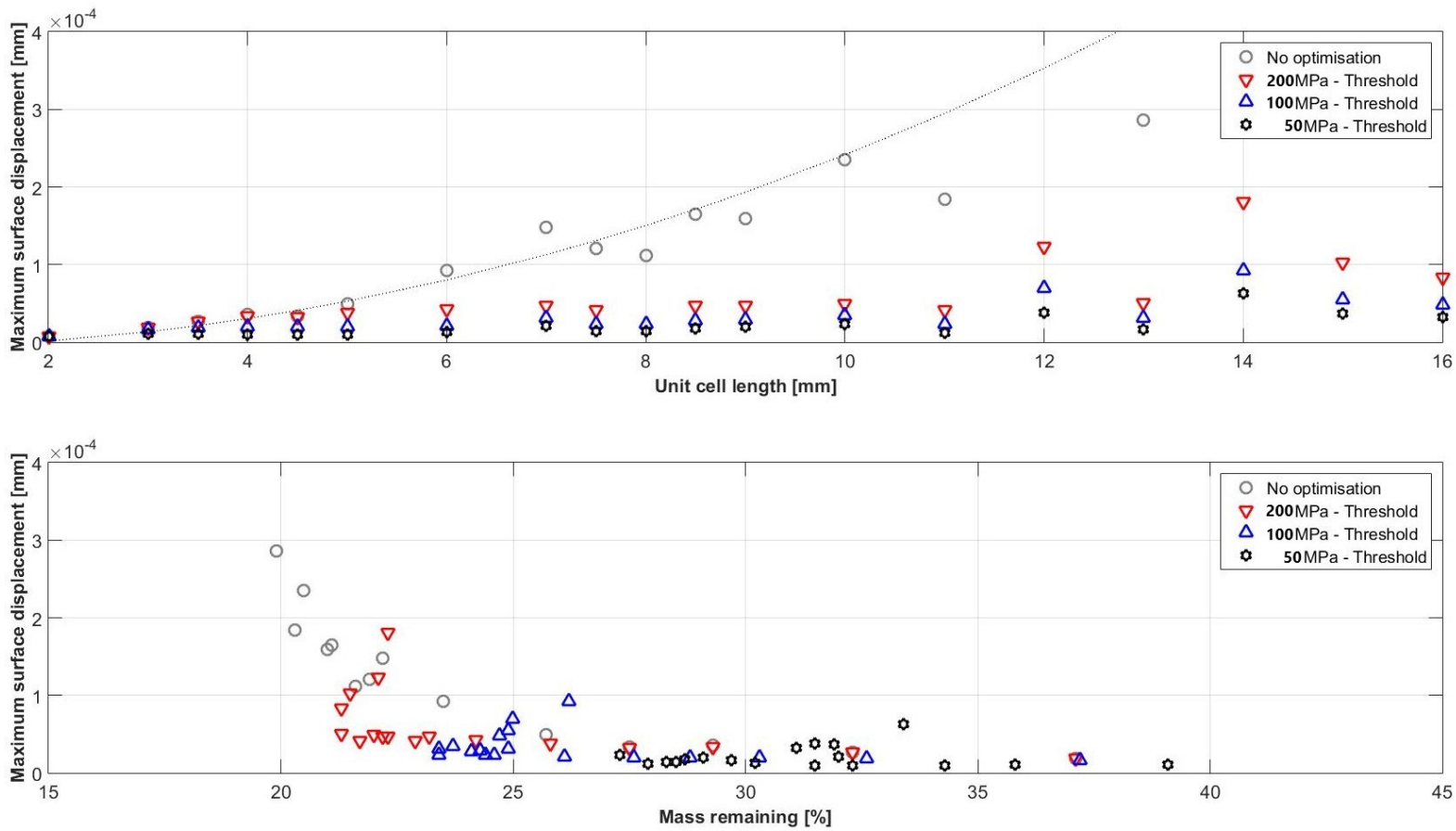

Figure 12. The effect of optimisation and unit length for a given threshold value. Upper plot - the maximum displacement recorded for each unit cell length for the three threshold values; lower plot - the maximum displacement as a function of mass remaining percentage. 


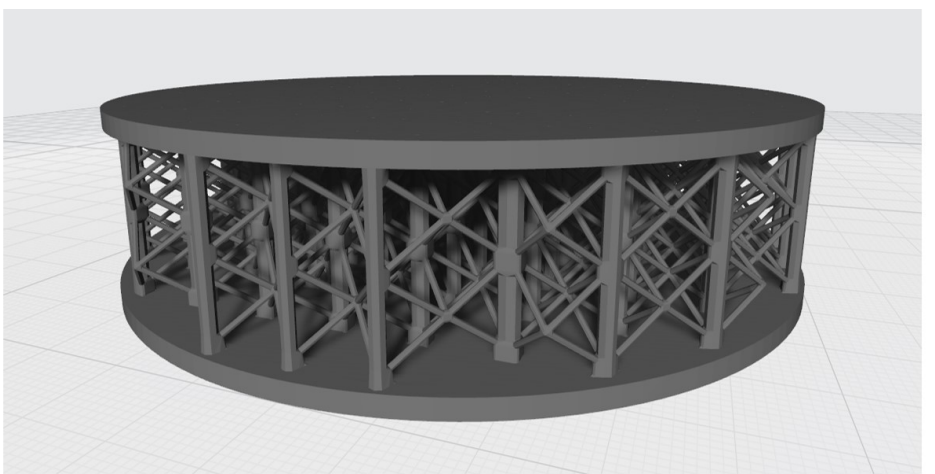

Optimised Star lattice mirror structure $(x, y, z)$

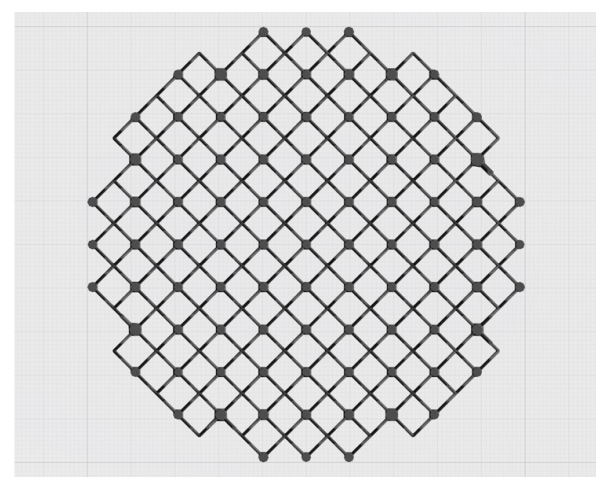

Optimised Star lattice $(x, y)$

Figure 13. Left - the optimised lattice mirror configuration with a unit cell length of $8 \mathrm{~mm}$ and with a $100 \mathrm{MPa}$ threshold value; right - a cross section of the lattice highlighting the increase of thickness of the lattice columns.

\subsubsection{Limitations of lattice generation}

The previous section described the challenge of fitting a cubic lattice to a cylindrical form, which is currently not supported within NetFabb. Therefore work has commenced at the University of Sheffield to generate a cylindrical lattice based upon increasing radii of nodal points as shown in Figure 14. A unit cell of the cylindrical lattice is similar to the $\mathrm{BCCz}$ lattice with vertical columns and $45^{\circ}$ diagonal struts, but a cell no longer has a cubic form and is a sector of an annulus. Development of the cylindrical lattice is on going with expected FEA simulations anticipated in the near future to assess its performance.

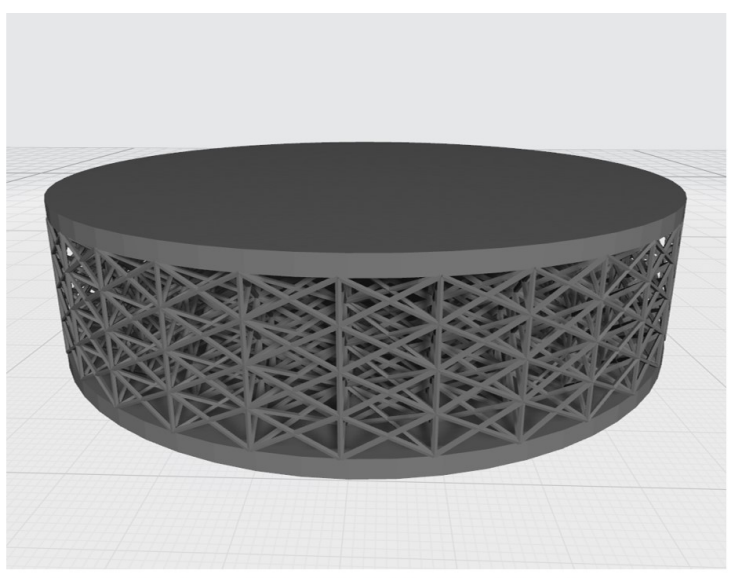

Rotationally symmetric BCCz lattice approximation

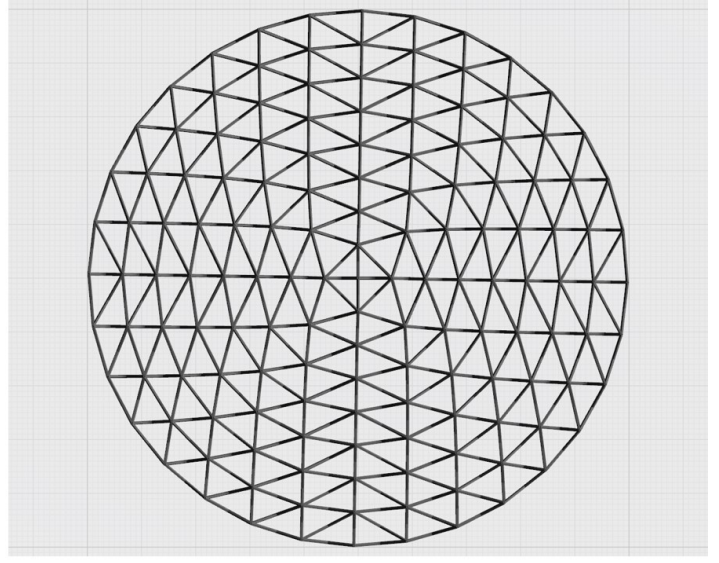

Lattice cross-section $(x, y, z)$

Figure 14. Left - a 3D representation of the cylindrical lattice incorporated within a sandwich mirror structure; right - a cross section through the lattice highlighting the configuration of the nodes and support struts.

\subsection{Honeycomb design}

Structures in nature are ideal for implementation within design for AM as they demonstrate an optimal design through evolution and the honeycomb of a bee is a prime example in the use of 'closest packing' within nature. A honeycomb is a construction that offers the largest volume (honey) for the minimum structure (beeswax) and it is created from two sets of hexagonal prisms ending in three rhomboidal faces placed back-to-back and offset to create a perfect tessellation, ${ }^{20}$ as shown in Figure 15. A approximation to the honeycomb design has been an inspiration for several telescope mirror geometries ${ }^{21,22}$ as well as mirror lightweighting structures $;^{23,24}$ however, applying the exact honeycomb cell geometry is challenging within traditional machining. The objective of this 
study is to apply the honeycomb construction that is seen in nature to create a lightweight sandwich mirror which exhibits two solid face plates and a rhombic dodecahedron interior.

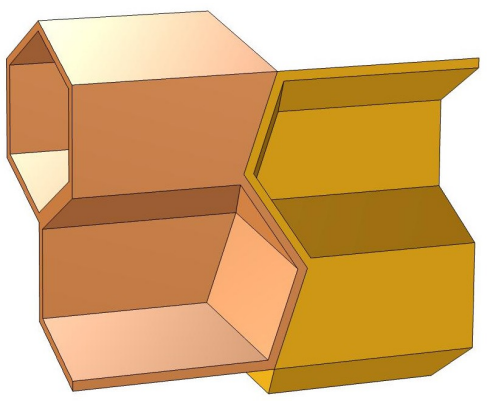

Figure 15. The honeycomb of a bee is created via two truncated tessellating rhombic dodecahedra.

To evaluate the rhombic dodecahedron pattern a series of unit cells were created with varying lightweighting ratios - as shown in Figure 16. In the case of honeycomb in nature, the rhombic dodecahedrons are form two layers as demonstrated in Figure 15; however, for simplicity, a single cell is modelled with a flat face top and bottom. These unit cells are then be tessellated to create the lightweight structure, as shown in Figure 17; however, like the lattices described in Section 3.4, the tessellated geometry does not fit a circular mirror.
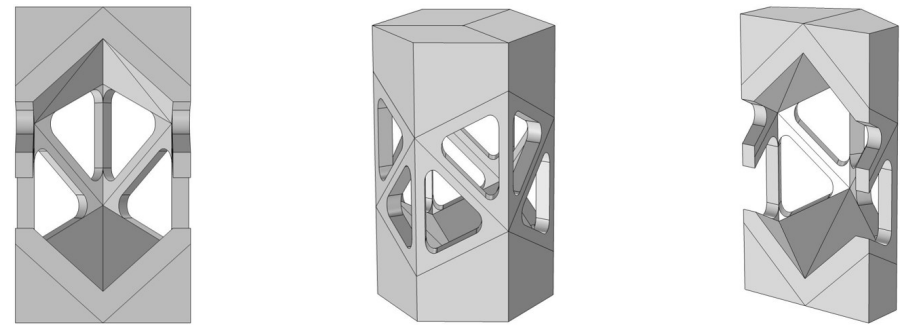

Figure 16. An illustration of the rhombic dodecahedron cell used in this study. The internal cavity measures $14.4 \mathrm{~mm}$ vertically (peak to trough), $8 \mathrm{~mm}$ horizontally (wall to wall) and with a wall thickness of $1 \mathrm{~mm}$. The cell measures $10 \mathrm{~mm}$ between the parallel hexagon edges.
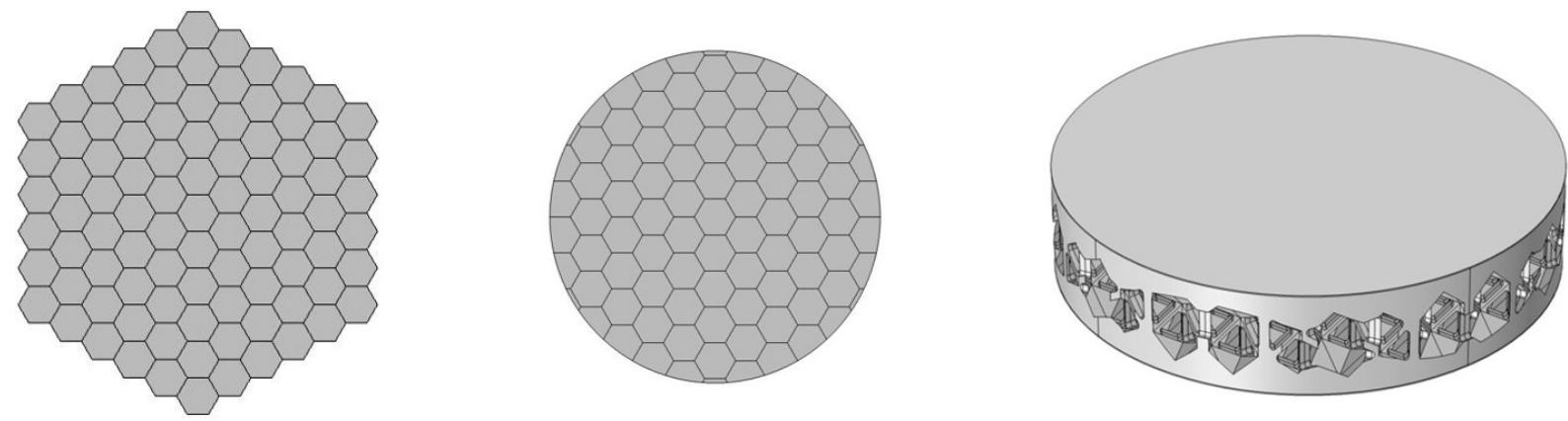

Figure 17. The tessellation of the hexagonal unit cells to create the mirror structure. Left - the tessellated units; middle - a cylinder cut from the units; and right - the mirror structure comprised of the hexagonal cells.

\subsubsection{Evaluation of a uniform lattice}

The first iteration of models investigated the use of a single unit cell of a given mass fraction. Three lightweighted cells were studied: 0.9 reduction, 0.8 reduction, and 0.7 reduction - these fractions refer to a scaled volume of a 
rhombic dodecahedron subtracted from the unit cell (as depicted in Figure 16), the additional polyhedra that create the flat top and bottom of the cells are not included in the assignment of reduction value.

The boundary conditions and the load applied are identical to those in the TO and lattice generation studies. The results from the uniform cells are presented in the upper half of Table 5. The results demonstrate that for $\sim 20 \%$ less mass an equivalent surface distortion is observed to the benchmark model, or conversely, for the equivalent mass remaining $\%$ the distortion is reduced.

\subsubsection{Evaluation of a non-uniform lattice}

Sections 3.3 and 3.4 demonstrated the advantage of optimisation routines to generate optimal structures and the same concept was applied to the honeycomb lattice manually. Using the optimised geometry generated in Figure 8 for no walls and with a vol. frac $=0.4$ as a template, the unit cells approximately within the TO geometry were changed to cells with a greater mass - i.e. lower mass reduction value. Figure 18 highlights how difference unit cells have been combined to incorporate TO within the honeycomb model.

There were four iterations of the model, each of which iterated upon the results gained from the previous model. The combinations of the trialled unit cells are presented in the table within Figure 18 and model 004 represents the final iteration. The performance of each of the models is presented in the lower half of Table 5; Model 004 demonstrates the reduction in distortion that can be achieved, in comparison to the uniform lattice, by altering the mass reduction of each of the cells.

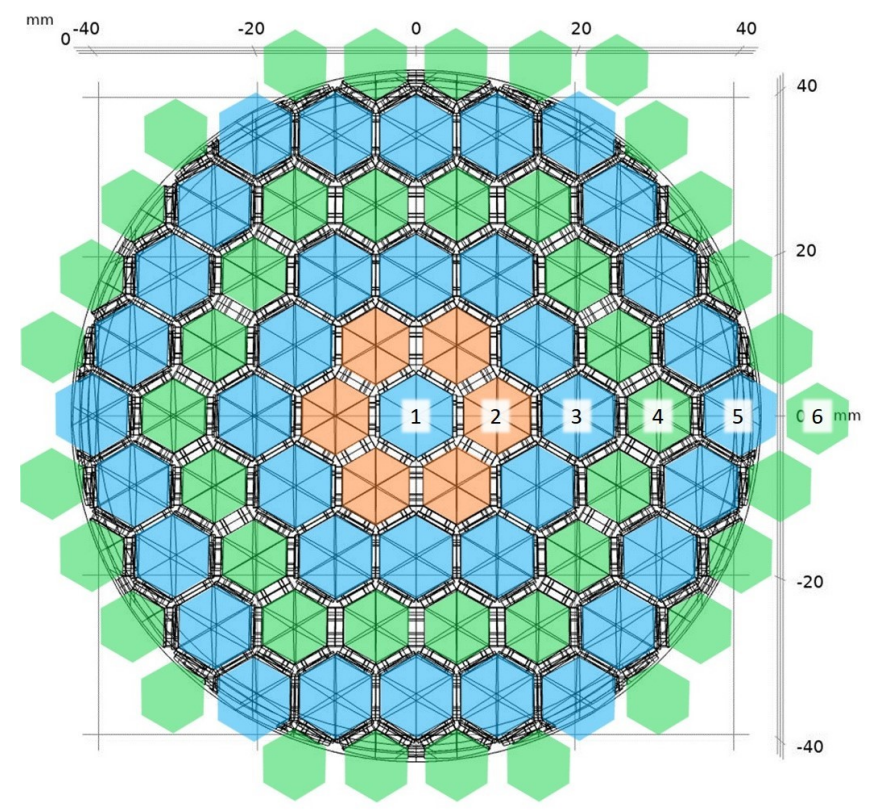

\begin{tabular}{|l|l|l|l|l|l|l|}
\hline \multicolumn{7}{|c|}{ The non-uniform honeycomb unit cell configuration } \\
\hline Model iteration & Cell 1 & Cell 2 & Cell 3 & Cell 4 & Cell 5 & Cell 6 \\
\hline 001 & 0.9 & 0.75 & 0.9 & 0.9 & 0.75 & 0.9 \\
\hline 002 & 0.9 & 0.75 & 0.9 & 0.75 & 0.9 & 0.9 \\
\hline 003 & 0.9 & 0.8 & 0.9 & 0.8 & 0.9 & 0.8 \\
\hline 004 & 0.9 & 0.825 & 0.9 & 0.8 & 0.9 & 0.8 \\
\hline
\end{tabular}

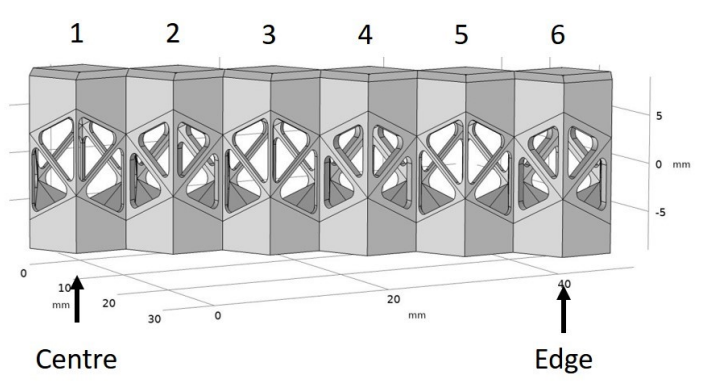

Figure 18. Left - a cross section of the Optimisation 004 highlighting the unit cells combinations applied within the honeycomb lattice; blue $=0.9$ reduction, orange $=0.825$ reduction, and green $=0.8$ reduction. Top right - the four lattice variations trialled to improve the performance of the lightweighting. Bottom right - an example of the six cells and labelled in reference to the image on the left.

The results presented in Table 5 have demonstrated the potential of more accurately representing designs seen in nature within mirror lightweighting. Combining the lattice with a topology optimisation solutions can create lightweighting that is more optimised to its function than a uniform lattice. These results represent a first step and there is significant scope to expand the study to evaluate different size unit cells, multiple layers of cells and adding a uniform edge to the mirror. 
Table 5. Honeycomb lattice simulations: mass reduction and surface distortion.

\begin{tabular}{|l|l|l|l|}
\hline Mirror design & Mass remaining \% & Distortion PV & Distortion RMS \\
\hline Honeycomb R0.9 uniform & 46.0 & $8.3 \mathrm{~nm}$ & $2.10 \mathrm{~nm}$ \\
Honeycomb R0.8 uniform & 57.5 & $2.3 \mathrm{~nm}$ & $0.54 \mathrm{~nm}$ \\
Honeycomb R0.7 uniform & 68.9 & $1.1 \mathrm{~nm}$ & $0.26 \mathrm{~nm}$ \\
\hline \hline Honeycomb + TO 001 & 53.6 & $6.3 \mathrm{~nm}$ & $1.9 \mathrm{~nm}$ \\
Honeycomb + TO 002 & 52.4 & $8.3 \mathrm{~nm}$ & $2.1 \mathrm{~nm}$ \\
Honeycomb + TO 003 & 51.2 & $2.6 \mathrm{~nm}$ & $0.5 \mathrm{~nm}$ \\
Honeycomb + TO 004 & 50.9 & $2.6 \mathrm{~nm}$ & $0.3 \mathrm{~nm}$ \\
\hline
\end{tabular}

\subsection{Evaluation of design optimisation process}

Within Section 3 a series of alternative approaches have been presented in regard to mirror lightweighting for the AM M1 CCAM mirror. Table 6 highlights a selection from each approach and how they compare against the FEA benchmark model of the manufactured design. Each method: TO, lattice generation, and honeycomb, highlight how different approaches are effective in reducing the weight of the mirror without compromising the distortion from the print-through effect.

Table 6. Comparison of the design optimisation results and the benchmark design.

\begin{tabular}{|l|l|l|l|}
\hline Mirror & Mass remaining \% & distortion PV & distortion RMS \\
\hline Benchmark design & 68.7 & $10.5 \mathrm{~nm}$ & $3.2 \mathrm{~nm}$ \\
\hline Topology optimisation & & & \\
No walls, 0.4 & 55.5 & $9.2 \mathrm{~nm}$ & $2.0 \mathrm{~nm}$ \\
No walls, 0.6 & 70.3 & $2.6 \mathrm{~nm}$ & $0.59 \mathrm{~nm}$ \\
\hline Lattice generation & & & \\
Star, unit cell 4 mm, no opt. & 29.3 & $35.9 \mathrm{~nm}$ & \\
Icosahedron, unit cell 4 mm, no opt. & 40.9 & $35.8 \mathrm{~nm}$ & \\
Tetra, unit cell 4 mm, no opt. & 32.9 & $72.5 \mathrm{~nm}$ & \\
Star, unit cell 4 mm, opt. at 50 MPa & 34.3 & $10.0 \mathrm{~nm}$ & \\
Star, unit cell 4 mm, opt. at 100 MPa & 30.3 & $19.6 \mathrm{~nm}$ & \\
Star, unit cell 4 mm, opt. at 200 MPa & 29.3 & $33.5 \mathrm{~nm}$ & \\
\hline Honeycomb & & & \\
Honeycomb R0.9 uniform & 46.0 & $8.3 \mathrm{~nm}$ & $2.1 \mathrm{~nm}$ \\
Honeycomb R0.7 uniform & 68.9 & $1.1 \mathrm{~nm}$ & $0.26 \mathrm{~nm}$ \\
Honeycomb + TO 004 & 50.9 & $2.6 \mathrm{~nm}$ & $0.3 \mathrm{~nm}$ \\
\hline
\end{tabular}

Topology optimisation is a powerful tool to indicate the locations to prioritise in terms of rigidity; however, arguably, it is best used in conjunction with another lattice structure to ensure a more uniform support of the mirror surface rather than a minimal number of regions with support or void. The lattice generation using NatFabb demonstrates how commercial AM software can be implemented in the use of mirror lightweighting and, despite the challenge of fitting a unit cell within a circular mirror, positive results can be achieved. In addition, the optimisation capacity of the software to add material to ensure structural integrity is highly beneficial to improve the performance of the lattice. The honeycomb lightweighting demonstrates how structures in nature can be implemented within AM design leading to improvements in lightweighting and surface displacement.

However, these initial concept structures are the first steps in the redesign process, as an ability to print, machine and mount the design is paramount. Printing and machining both impose restrictions on structures 
and therefore the optimum design is one that takes into account all these considerations. Furthermore, there are numerous other combinations, permutations and structures that are open to study in mirror lightweighting and this initial study details only a small subset of the options available.

\section{SUMMARY AND FUTURE WORK}

This paper has discussed the design logic for the fabricated AM mirror described in the accompanying paper Atkins et al. $2019^{12}$ and three different routes that could be applied in the future to optimise the design in terms of lightweighting percentage and form error. One of the key challenges in adopting AM within mirror fabrication is understanding the new production chain and the need to consider design requirements, AM design rules, AM fabrication and traditional machining, at the onset of the project to ensure a successful and innovative component (Section 2).

The design optimisation study (Section 3) demonstrates the benefits of computation optimisation, as well as trialling structures previously not considered. Due to the rapid turn-around in the fabrication of the benchmark design, the benefit of the optimised designs could not be implemented; however, these new designs are now ready for further development. In future work, the optimised designs will be considered from a printing and a machining view point, with additional features added to facilitate function and machining; several design iterations are expected to achieve this.

Beyond the regular lattices discussed in Section 3, irregular lattices, such as Voronoi cells, offer another option for mirror lightweighting where the cell size can be altered to optimise the structural performance. In addition to lightweighting, one of the primary benefits of AM is parts consolidation - i.e. the creation of 1 object from multiple parts. In relation to the CCAM CubeSat mirror, this approach could be applied to integrate the mirror with the mounting to the CubeSat chassis. Parts consolidation is appealing to minimise interfaces and retain identical material properties throughout a part. However, like design for AM, parts consolidation does require an holistic design approach to ensure the consolidated parts can be AM fabricated and machined.

\section{ACKNOWLEDGMENTS}

The authors acknowledge the funding received by the UK Space Agency through the National Space Technology Programme Pathfinder grant: NSTP3-PF2-008. Appreciation goes to Iain Todd at University of Sheffield; Mat Beardsley and Mike Harris at RAL Space for their assistance in this project. Cyril Bourgenot would like to acknowledge EPSRC for support with the grant EP/S001727/1.

\section{REFERENCES}

[1] Hilpert, E., Hartung, J., Risse, S., Eberhardt, R., and Tünnermann, A., "Precision manufacturing of a lightweight mirror body made by selective laser melting," Precision Engineering 0141-6359 (2018).

[2] Sweeney, M., Acreman, M., Vettese, T., Myatt, R., and Thompson, M., "Application and testing of additive manufacturing for mirrors and precision structures," Proc. SPIE 9574, 957406-957406-13 (2015).

[3] Herzog, H., Segal, J., Smith, J., Bates, R., Calis, J., De La Torre, A., Kim, D. W., Mici, J., Mireles, J., Stubbs, D. M., and Wicker, R., "Optical fabrication of lightweighted 3d printed mirrors," Proc. SPIE $\mathbf{9 5 7 3 ,}$ 957308-957308-15 (2015).

[4] Hilpert, E., Hartung, J., von Lukowicz, H., Herffurth, T., and Heidler, N., "Design, additive manufacturing, processing, and characterization of metal mirror made of aluminum silicon alloy for space applications," Optical Engineering 58(9) (2019).

[5] Mici, J., Rothenberg, B., Brisson, E., Wicks, S., and Stubbs, D. M., "Optomechanical performance of 3dprinted mirrors with embedded cooling channels and substructures," Proc.SPIE 9573, 9573 - 9573 - 14 (2015).

[6] Hu, R., Chen, W., Li, Q., Liu, S., Zhou, P., Dong, Z., and Kang, R., "Design optimization method for additive manufacturing of the primary mirror of a large-aperture space telescope," Journal of Aerospace Engineering 30(3), 04016093 (2017).

[7] Liu, J. and Jiang, B., "Topology optimization design of a space mirror," Proc. SPIE 9795, 97952Y-97952Y$10(2015)$. 
[8] Qu, Y., Wang, W., Liu, B., and Li, X., "Topology optimization design of space rectangular mirror," Proc. SPIE 10154, 1015421-1015421-7 (2016).

[9] Vukobratovich, D., [Optomechanical Engineering Handbook - Chapter 5: Lightweight Mirror Design], CRC Press LLC (1999).

[10] Schwertz, K. and Burge, J. H., [Field Guide to Optomechanical Design and Analysis], CRC Press LLC (2012).

[11] Roulet, M., Atkins, C., Hugot, E., Lemared, S., Lombardo, S., and Ferrari, M., "3d printing for astronomical mirrors," Proc.SPIE 10675, 10675 - 10675 - 13 (2018).

[12] Atkins, C., Brzozowski, W., Dobson, N., Milanova, M., Todd, S., Pearson, D., Brooks, D., Bourgenot, C., Snell, R. M., Sun, W., Cooper, P., Alcock, S. G., and Nistea, I.-T., "Additively manufactured mirrors for cubesats," Proc. of SPIE 11116-41 (2019).

[13] Allthorpe-Mullis, E., Brzozowski, W., Easdown, W., Feore, D., Grainger, W., Greenland, S., Hodgkins, M., Milanova, M., Payne, S., Pearson, D., Rowan, C., and Todd, S., "Cubesat camera: A low cost imaging system for cubesat platforms," Proceedings of iCubeSat 2018 (2018).

[14] Panesar, A., Abdi, M., Hickman, D., and Ashcroft, I., "Strategies for functionally graded lattice structures derived using topology optimisation for additive manufacturing," Additive Manufacturing 19, 81-94 (2018).

[15] Smith, C., Derguti, F., Nava, E. H., Thomas, M., Tammas-Williams, S., Gulizia, S., Fraser, D., and Todd, I., "Dimensional accuracy of electron beam melting (ebm) additive manufacture with regard to weight optimized truss structures," Journal of Materials Processing Technology 229, 128 - 138 (2016).

[16] Atkins, C., Feldman, C., Brooks, D., Watson, S., Cochrane, W., Roulet, M., Hugot, E., Beardsley, B., Harris, M., Spindloe, C., Alcock, S. G., Nistea, I.-T., Morawe, C., and Perrin, F., "Topological design of lightweight additively manufactured mirrors for space," Proc. of SPIE 10706 (2018).

[17] COMSOL, M., "Topology optimization of an mbb beam - v5.4." https://uk.comsol.com/model/topologyoptimization-of-an-mbb-beam-7428 (2018).

[18] Atkins, C., Feldman, C., Brooks, D., Watson, S., Cochrane, W., Roulet, M., Doel, P., Willingale, R., and Hugot, E., "Additive manufactured x-ray optics for astronomy," Proc.SPIE 10399, 10399 - 10399 - 15 (2017).

[19] Autodesk and NetFabb, "Lattice topologies." https://knowledge.autodesk.com/support/netfabb/learnexplore/caas/CloudHelp/cloudhelp/2017/ENU/NETF-Utility-Optimization/files/GUID-A738117A-64B14613-A904-FDB6130E2EDD-htm.html (2017).

[20] Pearce, P., [Structure in nature is a strategy for design], The MIT Press, fifth ed. (1990).

[21] Lightsey, P. A., Atkinson, C. B., Clampin, M. C., and Feinberg, L. D., "James webb space telescope: large deployable cryogenic telescope in space," Optical Engineering 51(1) (2012).

[22] Cayrel, M., Dierickx, P., Frster, A., Derie, F., Jochum, L., Pettazzi, L., Lucuix, C., Haupt, C., Mller, M., Vernet, E., and Pirard, J.-F., "Elt optomechanics: construction status," Proc. of SPIE 10700 (2018).

[23] Martin, H. M., Burge, J. H., Davis, J. M., Kim, D. W., Kingsley, J. S., Law, K., Loeff, A., Lutz, R. D., Merrill, C., Strittmatter, P. A., Tuell, M. T., Weinberger, S. N., and West, S. C., "Status of mirror segment production for the giant magellan telescope," Proc. of SPIE 9912 (2016).

[24] Rao, C., Gu, N., Zhu, L., Huang, J., Li, C., Cheng, Y., Liu, Y., Cao, X., Zhang, M., Zhang, L., Liu, H., Wan, Y., Xian, H., Ma, W., Bao, H., Zhang, X., Guan, C., Chen, D., and Li, M., "1.8-m solar telescope in china: Chinese large solar telescope," Journal of Astronomical Telescopes, Instruments, and Systems 1(2) (2015). 